\title{
ANÁLISIS DE LAS CAUSAS DE NO FUNCIÓN INICIAL DEL INJERTO EN NUESTRA SERIE DE LOS ÚLTIMOS ONCE AÑOS
}

\author{
R. BARRERO CANDAU, Mํ.P. PÉREZ ESPEJO, M.A. GENTIL GOVANTES*, \\ F.J. TORRUBIA ROMERO, N. CRUZ NAVARRO, J. LEAL ARENAS, \\ P. MONTAÑÉS MEDINA.
}

Unidad de Trasplante Renal. Servicio de Urología. *Servicio de Nefrología. H. U. Virgen del Rocío. Sevilla.

Actas Urol Esp. 27 (7): 524-529, 2003

\section{RESUMEN}

ANÁLISIS DE LAS CAUSAS DE NO FUNCIÓN INICIAL DEL INJERTO EN NUESTRA SERIE DE LOS ÚLTIMOS ONCE AÑOS

FUNDAMENTO: Conocer los factores de riesgo de disfunción inicial del injerto en nuestra serie. MATERIAL Y MÉTODO: Analizamos 469 trasplantes. 270 con buena función inicial y 199 sin función inicial. Las variables estudiadas en ambos grupos mediante estudio uni y multivariante fueron: edad, sexo y causa de muerte del donante, tipo de extracción y donde se realizó, lateralidad de la implantación, vasos múltiples, tiempos de isquemia fría, edad y sexo del receptor, compatibilidad HLA, retrasplante, Ac AntiVHC, PTH pretrasplante, años en lista de espera, hiperinmunización, número de transfusiones y tipo de inmunosupresión.

RESULTADOS: Estudio univariante: hubo diferencias significativas en la edad y causa de muerte del donante, tiempos de isquemia, años en lista de espera, hiperinmunización, número de transfusiones e incompatibilidad HLA-B. Estudio multivariante: hubo diferencias significativas en la edad del donante, tiempo de isquemia fría y de revascularización, e inmunosupresión cuádruple.

CONCLUSIONES: Los resultados aconsejan acortar en lo posible los tiempos de isquemia fría y de revascularización, y utilizar pautas inmunosupresoras menos nefrotóxica en casos de alto riesgo.

PALABRAS CLAVE: Análisis multivariante. Trasplante renal. Función renal retrasada.

\section{ABSTRACT \\ ANALYSIS OF THE REASONS FOR NON GRAFT INITIAL FUNCTION IN OUR SERIE OF THE LAST ELEVEN YEARS}

FUNDAMENT: To know which risk factor of delayed graft function in our patients.

MATERIAL AND METHODS: We analyzed 469 transplants, 270 had good initial function and 199 had delayed function graft. Variables studies in booth groups were: age, sex and dead cause of donant, type of extraction and place were it was done, implantation side, vases multiple, isquemia times, age and receptor sex, HLA compatibility, retransplant, Ac Anti-VHC, PTH pretransplant, years in waiting list, hiperinmunization, number of transfusion, and type of inmunosupretion.

RESULTS: Univariant study: significant differences were found in age and dead cause of donant, isquemia times, years in waiting list, hiperinmunization, number of transfusions, and HLA-B incompatibility. Multivariate study: we have significant differences in age of donant, could isquemia time, years in waiting list, ¿cuadruple? Inmunosupretion.

CONCLUTIONS: Results suggest to short could and revascularization isquemia time as possible, and use less nefrotoxic inmunosupretion pautes in high-risk patients.

KEY WORDS: Multivariante analyse. Renal transplant. Delayed graft function. 
$\mathrm{D}$ efinimos la disfunción inicial o no función inicial (NFI) del injerto como aquel trasplante renal (TR) que precisa diálisis en los primeros días tras el trasplante. Un injerto con NFI puede ser un riñón nunca funcionante (RNF) o un riñón con función renal retrasada (FRR). La diferencia entre ambos está en que el injerto con FRR llega a alcanzar en algún momento función suficiente para suspender la diálisis que precisó en los primeros días tras el trasplante (Ejemplo: necrosis tubular aguda), mientras que el RNF hace que el paciente vuelva a diálisis sin que el injerto haya funcionado nunca de forma suficiente para suspenderla.

Al observar la evolución de la función inicial de los injertos implantados en nuestro servicio a lo largo de los últimos años (Fig. 1), hemos comprobado que al igual que en la mayoría de los grandes registros de la UNOS y la UCLA, la NFI del injerto es la complicación postrasplante más frecuente que tenemos.

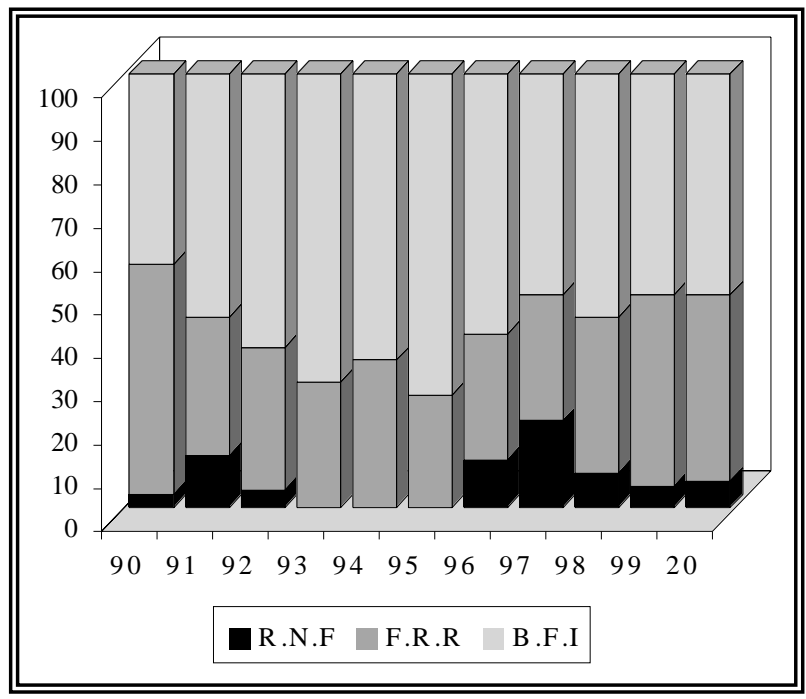

FIGURA 1. Evolución de la función inicial del trasplante renal en nuestro centro a lo largo de los últimos once años.

RNF = Riñón nunca funcionante.

$F R R=$ Función renal retrasada.

BFI = Buena función inicial.

Existen numerosos estudios acerca de las posibles causas o factores de riesgo de NFI del injerto (Tabla I), realizados unos en centros de referencia ${ }^{1-3,8-13}$ y otros basándose en las grandes bases de datos de los registros de pacientes ${ }^{4-}$ ${ }^{7}$, los cuáles tienen la virtud de amortiguar la

\section{TABLA I}

CAUSAS MÁS FRECUENTES DE DISFUNCIÓN INICIAL DEL INJERTO

\begin{tabular}{|lll|}
\hline Irreversibles & RNF \\
- Riñón no viable & \\
- Rechazo hiperagudo & \\
- Trombosis/Ateroembolismo & \\
- Etc. & FRR \\
Reversibles & \\
- Necrosis tubular aguda & \\
- Obstrucción/fuga urinaria & \\
- Rechazo agudo & \\
- Nefrotoxicidad por anticalcineurinas & \\
- Nefritis intersticial aguda (infección) & \\
- Etc. & \\
\hline
\end{tabular}

variación debida al llamado efecto centro. Pero este formidable cuerpo de evidencia no nos exime de analizar el problema en cada uno de nuestros hospitales, precisamente porque la definición de nuestro propio efecto centro, de las peculiaridades del fenómeno en nuestro contexto, será la mejor Guía para mejorar nuestra práctica.

El objetivo de este trabajo es precisamente conocer cuales de los factores de riesgo de NFI conocidos, que tienen mayor implicación en nuestra serie.

\section{MATERIAL Y MÉTODO}

De los 1.060 trasplantes realizados por nuestro grupo entre abril de 1978 y diciembre de 2002, hemos estudiado los 476 trasplantes renales de cadáver realizados en la década 19902000 , de los que se han podido estudiar 469 y se han excluido 7 en los que se realizó nefrectomía inmediata o precoz por ser la causa de fallo o pérdida del injerto obvia (neoplasia o infección del donante, problemas graves del órgano y problemas de compatibilidad). Realizamos un análisis retrospectivo de los pacientes trasplantados, que se dividieron en dos grupos entre los que se hicieron las comparaciones, uno con los TR que precisaron diálisis en los primeros días postrasplante (175) y otro (de 270) con los TR que no la precisaron por presentar buena función inicial (BFI), quedando excluidos del estudio los RNF (24). También se llevó a cabo un examen estratificado en 3 categorías: RNF, FRR y BFI: en casi todas las ocasiones hubo similitud de comportamiento entre los RNF y los riñones con FRR, lo que jus- 
tifica su tratamiento como una sola categoría. En los casos en que esto no ocurría, se hizo una comparación específica entre RNF y el resto de los casos.

La isquemia caliente o de extracción fue nula en la gran mayoría de los casos, puesto que generalmente se realizó perfusión in situ de los riñones; la mayor parte de los casos con isquemia caliente mayor de 0 obedecieron a paradas cardiacas del donante inmediatamente antes o durante la extracción. La isquemia fría se contabilizó desde el momento del clampaje del riñón tras la perfusión in situ en el cadáver hasta la extracción del riñón del hielo para el implante. La isquemia templada o de revascularización se midió desde ese momento hasta el desclampaje final de la arteria, incluyendo por tanto cualquier cirugía de banco o manipulación previa del órgano.

Respecto a la inmunosupresión empleada, hasta enero de 1997, la pauta de inmunosupresión aplicada en todos los casos fue una cuádruple secuencial: prednisona, azatioprina y globulina antitimocito (ATGAM), con introducción de ciclosporina $(\mathrm{CsA})$ a dosis baja $(3-6 \mathrm{mg} / \mathrm{Kg})$ al obtenerse función del injerto. En 1994, se modificó el esquema limitando la duración del tratamiento con globulina e introduciendo la CsA en todos los pacientes dentro de la primera semana, pero manteniendo las dosis iniciales bajas $1<8$ $\mathrm{mg} / \mathrm{Kg}$ ). Desde 1997 la inmunosupresión de base ha contenido mofetil micofenolato en la casi totalidad de los TR, asociado a CsA $(10 \mathrm{mg} / \mathrm{Kg})$ o tacrolimus $(0,2 \mathrm{mg} / \mathrm{Kg})$ desde el post-operatorio inmediato.

Nuestra sistemática de seguimiento de pacientes sin función inicial del injerto incluye una primera biopsia renal ante la sospecha de rechazo por la clínica o las exploraciones complementarias o, de forma rutinaria, si la disfunción se prolonga más de una semana. Posteriormente, la biopsia se repite si hay sospecha de rechazo o cada 7-10 días, hasta la reanudación de la función renal.

En cuanto al estudio estadístico, hemos realizado inicialmente estudio univariante, comparando en los dos grupos cada una de las variables que pueden modificar la función inicial del injerto, las variables estudiadas fueron las enumeradas en la Tabla II. Con aquellas variables en las
TABLA II

FACTORES DE RIESGO DE NFI ESTUDIADOS

\begin{tabular}{|l|l|}
\hline \multicolumn{1}{|c|}{$\begin{array}{c}\text { Relacionados con } \\
\text { Donante/ Técnica }\end{array}$} & \multicolumn{1}{c|}{$\begin{array}{c}\text { Relacionados con } \\
\text { Receptor }\end{array}$} \\
\hline - Edad & - Edad \\
- Sexo & - Sexo \\
- Causa de muerte & - Compatibilidad HLA \\
- Extracción local & - Años en lista de espera \\
- Tipo de extracción & - Hiperinmunización \\
- Lateralidad de la implantación & - Número de transfusiones \\
- Vasos múltiples, y & - Retrasplante \\
- Tiempos de isquemia & - Ac Anti-VHC \\
(fría y caliente) & - PTH pretrasplante \\
& - Tipo de inmunosupresión \\
\hline
\end{tabular}

que se obtuvo diferencias estadísticamente significativas, se realizó estudio multivariante para disminuir posibles sesgos de confusión y conocer la fuerza de asociación de cada variable teniendo en cuenta al resto de variables. Los test estadísticos empleados en el estudio según convino fueron la t de Student para las variables cuantitativas, la prueba de Mann-Withney (dicotómica independiente Vs. Cuantitativa $\mathrm{n}^{\circ}<30 \mathrm{u}$ ordinal) y la Chi-cuadrado para las variables cualitativas, mientras que el análisis multivariante empleado fue una regresión logística múltiple, paso a paso hacia delante.

\section{RESULTADOS}

En la Tabla III se muestran las variables, en relación con el donante y la técnica, en las que obtuvimos diferencias significativas en el estudio univariante. Podemos observar que los pacientes que precisaron diálisis por presentar disfunción inicial del injerto, eran aquellos en los que el injerto procedía de donantes de más edad, con muerte encefálica de origen vascular y extracción no multiorgánicas, además los injertos de pacientes con NFI presentaron mayores tiempos de isquemia fría y de revascularización. Por otro lado la NFI del injerto es más frecuente entre los receptores que están más años en lista de espera, tienen mayor incidencia de transfusiones y de hiperinmunización (Tabla IV). En cambio no apreciamos diferencias significativas respecto a sexo del donante, tipo de centro de extracción (local, en el centro de trasplante vs. procedente de otro hospital no trasplantador), tiempo de 
TABLA III

FACTORES RELACIONADOS CON EL DONANTE, EL ÓRGANO Y LA TÉCNICA ASOCIADOS A NFI DEL INJERTO EN EL ESTUDIO UNIVARIANTE

\begin{tabular}{||l|c|c|c||}
\hline Diálisis & Si & No & P \\
\hline Edad media donante (años) & 42,7 & 35,4 & $<0,01$ \\
\hline Causa muerte (\%) & & & \\
\hline TCE & 39 & 53 & $<0,03$ \\
\hline No TCE & 61 & 47 & $<0,02$ \\
\hline Extracción multiorgánica (\%) & 69,2 & 78,8 & $<0,02$ \\
\hline Isquemia fría (horas) & 22,3 & 20,0 & $<0,01$ \\
\hline Revascularización (min.) & 77,5 & 67,1 & $<0,02$ \\
\hline
\end{tabular}

TCE = Traumatismo craneoencefálico.

\section{TABLA IV}

FACTORES RELACIONADOS CON EL RECEPTOR ASOCIADOS A NFI

\begin{tabular}{|l|c|c|c|}
\hline Diálisis & Si & No & P \\
\hline Años en lista de espera & 5,2 & 3,8 & $<0,01$ \\
\hline Anticuerpos (PRA) >50\% & & & \\
\hline Máximo (\%) & 23,2 & 13,4 & $<0,01$ \\
\hline Actual (\%) & 5,4 & 1,6 & $<0,03$ \\
\hline № transfusiones & 9,3 & 5,6 & $<0,03$ \\
\hline Incomp. HLA-B & 1,32 & 1,17 & $<0,02$ \\
\hline
\end{tabular}

isquemia caliente, lado del riñón, número de arterias o venas y necesidad de cirugía de banco. Respecto a la inmunosupresión, no hubo diferencias en función del tipo de anticalcineurina (ciclosporina o tacrolimus) pero sí observamos una menor frecuencia de NFI con el uso de cual- quier tipo de anticuerpos. En concreto, con la pauta cuádruple secuencial de esteroides, ATG y azatioprina, con introducción diferida de ciclosporina, la incidencia de NFI resultó de 35,9\%, frente a $49,8 \%$ con el resto de las pautas.

La incidencia de RNF se asociaba en general a las mismas variables que el riñón con FRR, pero presentaba una tendencia a mayor frecuencia de extracción fuera de nuestro hospital y sobre todo en centros no trasplantadores $(41,7 \%$ vs $26,1 \%$, $\mathrm{p}=0,079$ ), y a mayor frecuencia de isquemia caliente superior a $0(25,0$ vs $14,0 \%, \mathrm{p}=0,12)$. También presentaban mayor proporción de casos con 2,3 ó 4 arterias $(29,2 ; 8,3$ y $4,2 \%$ frente a 18,$0 ; 2,3$ y $0,2 \%$ en el resto, $\mathrm{p}=0,003$ ) y con 2 ó 3 venas principales $(16,7$ y $4,2 \%$ frente 7,3 y $1,1 \%$, $\mathrm{p}=0,10$ ). Combinando lo anterior, encontramos que $58,3 \%$ de los RNF tenían arteria y/o vena múltiple frente a $25,6 \%$ del resto de los casos $(\mathrm{p}<0,001)$. Seguramente como consecuencia de lo anterior, 54,2 \% de los RNF precisaron cirugía de banco previa al TR frente a $23,8 \%$ del resto ( $\mathrm{p}=0,003)$.

Sin embargo en el estudio multivariante (Tabla V), realizado para evitar posibles sesgos de confusión y ver que variables tienen mayor fuerza de asociación, en un primer análisis mediante regresión logística, sólo aparecieron como factores pronósticos independientes la edad del donante, el tiempo de isquemia fría y el tiempo de revascularización.

A este modelo básico era posible incorporarle como factor predictivo adicional el año del trasplante (riesgo relativo 1.089 por año, IC 95\%: 1.004-1.181), es decir, que el riesgo de NFI aparentemente aumentaría a lo largo del período

\section{TABLA V}

ANÁLISIS MULTIVARIANTE

\begin{tabular}{|l|l|c|c|c|}
\hline Factor & Beta & P & O.R. & IC 95\% \\
\hline Edad del donante & 0,03 & 0,000 & 1,07 & $1,02-1,04$ \\
\hline Isquemia fría & 0,06 & 0,001 & 1,07 & $1,03-1,11$ \\
\hline Revascularización & 0,02 & 0,000 & 1,02 & $1,01-1,03$ \\
\hline Años en lista de espera & 0,08 & 0,002 & 1,08 & $1,03-1,13$ \\
\hline IS cuadruple & 0,67 & 0,002 & 1,95 & $1,27-3,00$ \\
\hline
\end{tabular}

IS = Inmunosupresión 
estudiado. Por otra parte, si se intentaba introducir el uso de inducción con anticuerpos como variable dicotómica (uso o no de cuádruple terapia), el nuevo modelo lo admitía como factor protector (la inmunosupresión sin inducción representaba un riesgo relativo de 1.95, con IC 95\%: 1.18-3.21), desplazando el factor tiempo y sin alterar sensiblemente la influencia del resto de los factores; el ajuste del modelo mejoraba. Estos hallazgos son de difícil interpretación. Por una parte, la incidencia de NFI descendió hasta 1995, para aumentar luego claramente (Fig. 1). Esta fase reciente de peor incidencia coincide con el abandono de la cuádruple terapia secuencial con globulinas antitimocito como pauta básica, debido a la introducción de los nuevos inmunosupresores. Pero también es cierto que la calidad del donante ha empeorado notablemente, al ampliarse los criterios de aceptación: los órganos proceden cada vez más de donantes de edad avanzada y de causa vascular; de hecho la edad del donante guarda una correlación positiva con el tiempo, débil pero significativa $(\mathrm{r}=0,17 ; \mathrm{p}<0,001)$. También es probable que las condiciones de los receptores sean ahora peores. En ambos aspectos es posible que se hayan dado cambios (a peor) no bien reflejados en las variables analizadas.

La repetición del análisis excluyendo a los RNF generaba un modelo prácticamente idéntico e incluso mejor ajustado. En cualquier caso la capacidad explicativa del modelo es muy limitada: solo clasifica correctamente el $71.2 \%$ de los casos.

\section{DISCUSIÓN}

Este análisis de las causas o factores de riesgo asociados a la disfunción inicial del injerto en nuestro centro ha confirmado la importancia de una serie de aspectos relativos al tipo de donante, al manejo del órgano y a las características del receptor que conducen a una mayor frecuencia de NFI. Es notable que el mayor estudio realizado en este terreno, el de Gjerston con más de 80.000 pacientes del registro de la U.N.O.S. ${ }^{7}$, atribuyera a las tres principales variables identificadas por nosotros (la edad del donante, los años en diálisis y el tiempo de isquemia fría) casi dos tercios de la variabilidad asignable en la incidencia de NFI (este estudio no analizaba el tiempo de revascularización).
Desde el punto de vista de la práctica médica es posible distinguir dos clases de factores influyentes en la disfunción inicial del injerto. Por un lado, ciertas variables como la edad del donante y la longevidad en lista de espera, que son en cierto modo externas al proceso de ejecución del TR y que dependen más bien de la estrategia de obtención de órganos y de los criterios de selección del receptor. El margen de mejora en este terreno está acotado de entrada por la limitación de donantes disponibles. De hecho, si redujéramos la edad de los donantes aceptados, aumentaríamos el tiempo de espera en diálisis (a no ser que redujéramos paralelamente la admisión de candidatos, lo que parece poco admisible).

De otra parte, hay factores más accesibles a la intervención. Así, hay una gran potencialidad de reducción de la NFI mediante el acortamiento de los tiempos de isquemia fría y de recalentamiento. Este último puede mejorarse a través del perfeccionamiento y acumulación de experiencia quirúrgica individual y de grupo: el TR debería ser una técnica reservada a cirujanos muy dedicados (es decir, un grupo reducido) y expertos.

Los tiempos de isquemia fría han sido prolongados en nuestra serie, probablemente por la falta de coordinación de los distintos estamentos (celadores, auxiliares, ATS, anestesistas, nefrólogos, y urólogos) implicados en la cadena necesaria para la realización de un TR, así como por la prioridad que se da a otras actividades como los trasplantes cardíaco, hepático, y a los quirófanos reglados de la mañana, en detrimento del TR. A raíz de este análisis se han tomado determinadas medidas en nuestro centro que ayudarán a disminuir la incidencia de NFI, entre las que se encuentra la reorganización del quirófano reglado de mañana siempre que se trate de riñones subóptimos, teóricamente con menor masa nefronal, para que éstos tengan el menor tiempo de isquemia fría posible. Otras medidas para acortar el tiempo de isquemia fría han sido: la prueba cruzada previa utilizando suero congelado de todos los posibles receptores, el sero que se almacena en la seroteca, se actualiza periódicamente. La mejora de la localización de receptores con los teléfonos móviles. Por otra parte hacen falta medidas para mejorar la coordinación de los diferentes eslabones implicados; la isquemia fría 
puede ser disminuida si cada uno de los eslabones que intervienen en el trasplante reconsidera su práctica $\mathrm{y}$, sobre todo, sus horarios de trabajo $^{13}$. Sin duda se va a requerir también una intervención decidida por parte de los gestores del hospital, asignando de forma prioritaria recursos materiales y humanos a esta misión. Finalmente, cabe la posibilidad de aminorar la frecuencia o gravedad da la NFI con el empleo de una inmunosupresión inicial menos nefrotóxica. Nuestra experiencia al respecto es algo equívoca, pero existen datos en la literatura que apoyan el retraso en la introducción y/o dosis iniciales menores de anticalcineurinas.

\section{REFERENCIAS}

1. GEDDES CC, COLE E, WADE J, CATTRAN D, FENTON S, ROBINETTE M, STEWART R, HEMMING A, CATTRAL M, GARCÍA A, CARDELLA CJ.: Factors influencing long-term primary cadaveric kidney transplantation-importance of functional renal mass versus avoidance of acute rejections-the Toronto Hospital experience 1985-1997. Clin Transpl 1998: 195-203.

2. MCLAREN AJ, JASSEM W, GRAY DW, FUGGLE SV, WELSH KI, MORRIS PJ.: Delayed graft function: risk factors and the relative effects of early function and acute rejection on long-term survival in cadave-ric renal transplantation. Clin Transplant 1999 jun; 13 (3): 266-272.

3. SALMELA K, KYLLONEN L.: Renal transplantation in Helsinki: influence on long-term survival of early postransplant factors. Clin Transpl 1999: 173-179.

4. OJO AO, WOLFE RA, HELD PJ, PORT FK, SCHMOUDER RL.: Delayed graft function: risk factors and implications for renal allograft survival. Transplantation 1997 apr 15; 63 (7): 968-974.

5. TEJANI AH, SULLIVAN EK, ALEXANDER SR, FINE RN, HARMON WE, KOHAUT EC.: Predictive factors for delayed graft function (DGF) and its impact on renal graft survival in children: a report of the North American Pediatric Renal Transplant Cooperative Study (NAPRTCS). Pediatr Transplant 1999 nov; 3 (4): 293-300.
6. MORESO F, SERON D, GIL-VERNET S, RIERA L, FULLADOSA X, RAMOS R, ALSINA J, GRINYO JM.: Donor age and delayed graft function as predictors of renal allograft survival in rejection-free patients. Nephrol Dial Transplant 1999 apr; 14 (4): 930-935.

7. GJERTSON DW.: Impact of delayed graft function and acute rejection on kidney graft survival. Clin Transpl 2000: 467-480.

8. LEHTONEN SR, ISONIEMI HM, SALMELA KT, TASKINEN EI, VON WILLEBRAND EO, AHONEN JP, VWILLEBRAND EO.: Long-term graft outcome is not necessarily affected by delayed onset of graft function and early acute rejection. Transplantation 1997 jul 15; 64 (1): 103-107.

9. MARCEN R, OROFINO L, PASCUAL J, DE LA CAL MA, TERUEL JL, VILLAFRUELA JJ, RIVERA ME, MAMPASO F, BURGOS FJ, ORTUNO J.: Delayed graft function does not reduce the survival of renal transplant allografts. Transplantation 1998 aug 27; 66 (4): 461-466.

10. PÉREZ FONTAN M, RODRÍGUEZ-CARMONA A, BOUZA P, VALDES F.: The prognostic significance of acute renal failure after renal transplantation in patients treated with cyclosporin. BJM 1998 jan; 91 (1): 27-40.

11. BOOM H, MALLAT MJ, DE FIJTER JW, ZWINDERMAN AH, PAUL LC.: Delayed graft function influences renal function, but not survival. Kidney Int 2000 aug; 58 (2): 859-866.

12. PFAFF WW, HOWARD RJ, PATTON PR, ADAMS VR, ROSEN CB, REED AI.: Delayed graft function after renal transplantation. Transplantation 1998 jan 27; 65 (2): 219-223.

13. ASDERAKIS A, DYER P, AUGUSTINE T, WORTHINGTON J, CAMPBELL B, JOHNSON RW.: Effect of cold ischemic time and HLA matching in kidneys coming from "young" and "old" donors: do not leave for tomorrow what you can do tonight. Transplantation 2001 aug 27; 72 (4): 674-678.

Dr. R. Barrero Candau

C/ Castillo Alcalá de Guadaira, 17

Portal C-2, 1ㅇ A

41013 Sevilla

e-mail: rafaelbarrero@latinmail.com

(Trabajo recibido el 7 febrero de 2003) 